Pacific Journal of Mathematics

LIE MAPPINGS IN CHARACTERISTIC

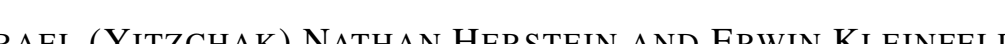




\section{LIE MAPPINGS IN CHARACTERISTIC 2}

\section{N. Herstein AND ERwin Kleinfeld ${ }^{1}$}

1. Introduction. In a previous paper [2] one of the authors proved that Jordan homomorphism, that is, an additive mapping $\varphi$ onto a prime ring of characteristic not 2 or 3 which preserves squares, is either a homomorphism or an anti-homomorphism. Smiley [6] then showed that this was also true in the characteristic 3 case; in the characteristic 2 case he showed that the same conclusion holds for $\phi$ if one assumes $\varphi(a b a)=\varphi(a) \varphi(b) \varphi(a)$ for all $a$ and $b$.

We concern ourselves here with mappings $\phi$ onto a simple ring of characteristic 2 which preserve commutators and cubes. This situation is of interest for in characteristic 2 Jordan homomorphisms are the same thing as Lie homomorphisms, that is, mappings which preserve commutators. Lie mappings for matrices have been completely determined [5]. However little information is known for general simple rings.

Of particular interest is the type of argument used to establish the result for it uses the theory of Lie and Jordan ideals and substructures of simple rings developed by Herstein [3].

2. Main section. As is customary the commutator $a b-b a$ will be denoted by $[a, b]$.

Initially $\rho$ will be a mapping from a simple ring $R$ onto a simple ring $R^{\prime} \neq 0$ of characteristic 2 which satisfies

$$
\begin{aligned}
\varphi(x+y) & =\varphi(x)+\varphi(y) \\
\mathcal{P}\left(z^{2}\right) & =\varphi(z)^{2} \\
\mathcal{P}\left(z^{3}\right) & =\varphi(z)^{3}
\end{aligned}
$$

for all $x, y, z \in R$. Later we weaken (ii) to the assumption that $\varphi(x y-y x)=\varphi(x) \varphi(y)-\varphi(y) \varphi(x)$.

Although we do not assume that the characteristic of $R$ is 2 , it can be easily proved. Clearly $\varphi(2 x)=0$ for all $x \in R$; but the kernel of $\varphi$ is a Jordan ideal of $R$, and if the characteristic of $R$ is not 2, the only non-zero Jordan ideal of $R$ would be $R$ itself [3]; thus $2 x=0$ for all $x \in R$ and $R$ has characteristic 2 .

Assume that $\rho$ is a mapping satisfying (i), (iii) and (ii)' $\rho(x y+y x)=$ $\varphi(x) \varphi(y)+\varphi(y) \varphi(x)$ for all $x, y \in R$, and that $R$ is a field. From (ii)', $R^{\prime}$ is a field. On linearizing (iii) we find that

(1) $\varphi(x y(x+y))=\varphi(x) \varphi(y) \varphi(x+y)$ for all $x, y \in R$. Let $W=$

Received July 30, 1959. The research of this paper was supported by an OOR contract with Cornell University. 
$\{z \in R \mid \varphi(z)=0\}$. Thus if $0 \neq z \in W$ from (1), for all $b \in R, \varphi(z b(z+b))=0$. Putting $b=z^{-1}$ in this yields $0=\varphi(z)=\varphi\left(z^{-1}\right)$. If we replace $b$ by $z^{-2}$ we obtain $\varphi(1)=\varphi\left(z^{-3}\right)=\varphi\left(z^{-1}\right)^{3}=\varphi(z)^{3}=0$. Thus putting $x=1$ in (1) leads to $\phi\left(b^{2}\right)=\varphi(b)$ for all $b \in R$. In (1), putting $x=b^{-2}$ we arrive at $\varphi\left(b^{-3}\right)=$ $\varphi\left(1+b^{-3}\right)=\phi\left(b^{-2}\right) \varphi(b)\left(\phi\left(b+b^{-2}\right)\right)$. Since $\phi\left(b^{-3}\right)=\varphi\left(b^{-1}\right)^{3}, \phi\left(b^{2}\right)=\varphi(b)$,

(2) $\varphi\left(b^{-1}\right)^{3}=\varphi\left(b^{-1}\right) \varphi(b) \varphi\left(b+b^{-1}\right)=\varphi\left(b+b^{-1}\right)$ from (1). By symmetry, $\varphi(b)^{3}=\varphi\left(b+b^{-1}\right)$, and so $\varphi\left(b^{3}\right)=\varphi(b)^{3}=\varphi\left(b^{-1}\right)^{3}=\varphi\left(b^{-3}\right)$, whence $\varphi\left(b^{3}+b^{-3}\right)=0$. Thus, using this in (2) we have that $\varphi\left(b^{-3}\right)^{3}=\varphi\left(b^{3}+b^{-3}\right)=0$, and so $\varphi\left(b^{-3}\right)=0$; thus $\varphi\left(b^{-1}\right)=0$ for all $b \in R$, that is, $\varphi(x)=0$ for all $x \in R$, forcing $R^{\prime}=0$. So we must assume that $\varphi(z)=0$ implies that $z=0$.

In (1) let $x \neq 0,1$ be arbitrary, $y=x^{-1}$. Then

$$
\varphi\left(x+x^{-1}\right)=\phi(x) \varphi\left(x^{-1}\right) \varphi\left(x+x^{-1}\right),
$$

and since $x+x^{-1} \neq 0$, we have that $\varphi(x) \varphi\left(x^{-1}\right)=1$. Since $0 \neq \varphi(1)=$ $\varphi\left(1^{3}\right)=\varphi(1)^{3}, \varphi(1)^{2}=1$, and since $R^{\prime}$ is a field of characteristic 2 , this forces $\varphi(1)=1$. Thus $\varphi\left(x^{-1}\right)=\varphi(x)^{-1}$ for all $x \neq 0 \in R$. By a result of Hua [4] $\varphi$ must be an isomorphism. If $R=G F(2)$, then since $\phi(0)=0$, $\varphi(1)=1$ it is trivial that $\varphi$ is an isomorphism. Therefore the special case that $R$ is a field is disposed of, and we assume henceforth in the paper that $R$ is a simple ring which is not a field.

Suppose that $R$ is simple and has a unit element and $\varphi$ satisfies (i), (ii)' and (iii). Suppose $\varphi(1)=a$. By (ii)', $a$ is in $Z^{\prime}$, the center of $R^{\prime}$. Also $a^{3}=\varphi(1)^{3}=\varphi\left(1^{3}\right)=a$, and so $a=0$ or $a^{2}=1$. If $a^{2}=1$, since $a$ is in $Z^{\prime}$, and $R^{\prime}$ is simple of characteristic $2, a=1$. Suppose that $a=$ $\varphi(1)=0$. Thus for all $x \in R, \varphi(x)^{3}=\varphi(x+1)^{3}=\varphi\left((x+1)^{3}\right)=\varphi\left(x^{3}+x^{2}+x+1\right)$. Thus $\varphi\left(x^{2}\right)=\varphi(x)$; replacing $x$ by $x+y$ in this yields $\varphi(x y+y x)=0$ for all $x, y \in R$, and so $R^{\prime}$ is a field. Now

$$
\begin{gathered}
\varphi\left(x^{3}+x^{2} y+x y x+y x^{2}+y^{2} x+y x y+x y^{2}+y^{3}\right)=\varphi\left((x+y)^{3}\right) \\
=(\varphi(x)+\varphi(y))^{3}=\varphi(x)^{3}+\varphi(x)^{2} \varphi(y)+\varphi(y)^{2} \varphi(x)+\varphi(y)^{3} .
\end{gathered}
$$

This leads, using $\varphi(a b+b a)=0$ to

$$
\varphi\left(x^{2} y+y^{2} x\right)=\varphi(x)^{2} \varphi(y)+\varphi(y)^{2} \varphi(x) \text { for all } x, y \in R .
$$

In this replace $x$ by $x+z$. A simple computation then shows that $\varphi((x z+z x) y)=0$ for all $x, y, z \in R$. From this we get that $\varphi(R(x z+z x) R)=0$ for all $x, z \in R$; if $x z+z x \neq 0$ by the simplicity of $R, R(x z+z x) R=R$. Since $R^{\prime} \neq 0$ we are forced to assume that $x z+z x=0$ for all $x, z \in R$. But then $R$ is a field and from the case already disposed of we know that $\varphi$ is an isomorphism and so $\varphi(1)=1$, contradicting $\varphi(1)=0$. Thus we have shown that if $\varphi$ is an additive mapping preserving cubes and commutators and if $R$ has a unit element, then $\varphi(1)=1$. Now considering $\varphi(x+1)^{3}$ we readily see that $\varphi\left(x^{2}\right)=\varphi(x)^{2}$. Thus (ii)' and (iii) imply 
(ii). Thus it would suffice in this case to assume that $\phi$ preserves cubes and commutators.

We begin with

Lemma 1. For all $x, y \in R$,

$$
\varphi(x y x+y x y)=\varphi(x) \varphi(y) \varphi(x)+\phi(y) \varphi(x) \varphi(y) .
$$

Proof. Linearizing (ii) it is clear that $\varphi(a b+b a)=\mathscr{\rho}(a) \mathcal{P}(b)+\varphi(b) \varphi(a)$. In (iii) replace $z$ by $x+y$. Then

$$
\begin{aligned}
& \varphi\left(x^{3}+x^{2} y+x y x+y x^{2}+y^{2} x+y x y+x y^{2}+y^{3}\right)=\varphi(x)^{3}+\varphi(x)^{2} \varphi(y) \\
& \quad+\varphi(x) \varphi(y) \varphi(x)+\varphi(y) \varphi(x)^{2}+\varphi(y)^{2} \varphi(x)+\varphi(y) \varphi(x) \varphi(y) \\
& \quad+\varphi(x) \varphi(y)^{2}+\varphi(y)^{3} .
\end{aligned}
$$

Because of (iii) $\varphi\left(x^{3}\right)=\mathscr{P}(x)^{3}, \mathcal{P}\left(y^{3}\right)=\varphi(y)^{3}$. Also from (ii) and its linearized form, $\varphi\left(x^{2} y+y x^{2}\right)=\varphi(x)^{2} \varphi(y)+\varphi(y) \varphi(x)^{2}$ and $\rho\left(y^{2} x+x y^{2}\right)=$ $\varphi(y)^{2} \varphi(x)+\varphi(x) \varphi(y)^{2}$. Substituting these results into the above linearized form of (iii) we obtain the result of the lemma.

Lemma 1 will now be strengthened to

Lemma 2. For all $x, y, z \in R$,

$$
\varphi(x y z+z y x)=\varphi(x) \varphi(y) \varphi(z)+\phi(z) \varphi(y) \varphi(x) .
$$

Proof. In the result of lemma 1 replace $x$ by $x+z$. Carrying out the linearization and using Lemma 1 on the resulting expressions we are left with the expression in Lemma 2.

Lemma 3. If $\varphi(z)=0$ then $z=0$.

Proof. Suppose $\varphi(z)=0$. From Lemma 2 it follows that

$$
\varphi(x y z+z y x)=\varphi(x) \varphi(y) \rho(z)+\varphi(z) \mathcal{P}(y) \mathcal{P}(x)=0
$$

for all $x, y \in R$.

However, $\varphi(x y z+z x y)=\varphi(x y) \varphi(z)+\varphi(z) \varphi(x y)=0$. Adding these two to we obtain $\varphi(z(x y+y x))=0$ for all $x, y \in R$. Letting $z^{\prime}=z(x y+y x)$ and repeating the argument used above, $\varphi\left(z^{\prime}(u v+v u)=0\right.$, that is, $\varphi(z(x y+y x)(u v+v u))=0$. Continuing in this manner, $\varphi(z \alpha)=0$ for all $a$ in the subring generated by all the elements $x y+y x$ with $x, y \in R$. Since $R$ is simple and not a field, by a result of Herstein [3], the subring generated by all the $x y+y x$ is $R$ itself. Thus $\varphi(z R)=0$. By a symmetrical argument, $\varphi(R z)=0$. But then, replacing $z$ by $R z$ we obtain that $\varphi(R z R)=0$. If $z \neq 0$, by the simplicity of $R, R z R=R$, and so 
$\varphi(R)=0$, whence $R^{\prime}=0$, contrary to assumption. Hence we can conclude that $z=0$.

We now prove

Lemma 4. For all $x, y \in R \varphi(x y x)=\varphi(x) \varphi(y) \varphi(x)+\lambda$ where $\lambda$ commutes with $\varphi(x)$ and with $\varphi(y)$.

Proof. Since $x^{2} y x+x y x^{2}=x(x y x)+(x y x) x$ we know that $\phi\left(x^{2} y x+x y x^{2}\right)=$ $\varphi(x) \varphi(x y x)+\varphi(x y x) \varphi(x)$. But, on the other hand, by Lemma 2

$$
\varphi\left(x^{2} y x+x y x^{2}\right)=\varphi(x)^{2} \varphi(y) \varphi(x)+\varphi(x) \varphi(y) \varphi(x)^{2} .
$$

Equating these two expressions it follows that

$$
\varphi(x)(\varphi(x y x)+\varphi(x) \varphi(y) \varphi(x))=(\varphi(x y x)+\varphi(x) \varphi(y) \varphi(x)) \varphi(x) .
$$

From Lemma 1,

$$
\varphi(y x y)+\varphi(y) \varphi(x) \varphi(y)=\varphi(x y x)+\varphi(x) \varphi(y) \varphi(x)
$$

and so it also commutes with $\phi(y)$, proving the lemma.

Of central importance in our subsequent arguments is the

Lemma 5. If $x y=y x$ then $\varphi(x y)=\varphi(x) \varphi(y)$.

Proof. Note that $0=\varphi(0)=\varphi(x y+y x)=\varphi(x) \varphi(y)+\varphi(y) \varphi(x)$, $\varphi(x) \varphi(y)=\varphi(y) \varphi(x)$.

From Lemma 2 we have that

$$
\varphi(x y z+z y x)=\varphi(x) \varphi(y) \varphi(z)+\varphi(z) \varphi(y) \varphi(x),
$$

while from our assumptions on $\varphi, \varphi(x y z+z x y)=\varphi(x y) \varphi(z)+\phi(z) \varphi(x y)$. Since $z x y=z y x$, we deduce that $\rho=\varphi(x y)+\varphi(x) \varphi(y)$ has the property that $[\rho, \varphi(z)]=0$ for all $z$.

On the other hand, $x(x y)=(x y) x$, and so by a similar argument $\sigma=\varphi\left(x^{2} y\right)+\varphi(x) \varphi(x y)$ has the property that $[\sigma, \varphi(z)]=0$. Moreover, since $x^{2}$ and $y$ commute, by the same argument as above $\varphi\left(x^{2} y\right)=$ $\varphi(x)^{2} \varphi(y)+\tau$ where $[\tau, \varphi(z)]=0$. Thus

$$
\sigma=\tau+\varphi(x)(\varphi(x y)+\varphi(x) \varphi(y))=\tau+\varphi(x) \rho .
$$

But then $[\varphi(x) \rho, \varphi(z)]=0$ and so $[\varphi(x), \varphi(z)] \rho=0$. Since $\rho$ is in the center of $R^{\prime}$ and $R^{\prime}$ is a prime ring we must have either $\rho=0$ or $[\varphi(x), \varphi(z)]=0$. If $\rho=0$ we have proved our contention. If $\rho \neq 0$, $[\varphi(x), \varphi(z)]=0$ for all $z$, and so $\varphi[x, z]=0$. Using Lemma 3, we deduce that $[x, z]=0$, hence $x$ is in the center of $R$. Similary $y$ is in the center of $R$. Since $R$ is not a field there is an element $w$ not in the center of $R$. Since $x$ is in the center, $x w=w x$. As we have just seen, 
either $\phi(x w)=\phi(x) \phi(w)$ or both $x$ and $w$ are in the center of $R$. Since $w$ is out of the center, $\varphi(x w)=\varphi(x) \varphi(w)$. Since $y$ is in the center of $R, y+w$ is not in the center so

$$
\begin{aligned}
& \mathcal{P}(x y)+\mathcal{P}(x) \varphi(w)=\varphi(x y)+\mathcal{P}(x w)=\mathscr{P}(x y+x w) \\
& \quad \mathcal{P}(x(y+w))=\mathcal{P}(x) \mathcal{P}(y+w)=\mathscr{P}(x) \varphi(y)+\varphi(x) \mathcal{P}(w) .
\end{aligned}
$$

Thus $\varphi(x y)=\rho(x) \varphi(y)$, and so $\rho=0$, contrary to the assumption that $\rho \neq 0$. Thus in any case we conclude that $\rho=0$ and so that $\mathcal{P}(x y)=$ $\varphi(x) \mathcal{P}(y)$, thereby proving the lemma.

LEMma 6. If the center of $R$ contains an element $\lambda \neq 0,1$ then $\varphi(x y x)=\varphi(x) \varphi(y) \varphi(x)$ for all $x, y \in R$.

Proof. Substituting $\lambda x$ for $x$ in Lemma 1 we see that

$$
\varphi\left(\lambda^{2} x y x+\lambda y x y\right)=\varphi(\lambda x) \varphi(y) \varphi(\lambda x)+\phi(y) \mathcal{P}(\lambda x) \varphi(y) .
$$

In light of Lemma $5, \rho\left(\lambda^{2} x y x\right)=\varphi(\lambda)^{2} \varphi(x y x)$,

$$
\varphi(\lambda x)=\varphi(\lambda) \mathcal{P}(x) \text { and } \varphi(\lambda y x y)=\varphi(\lambda) \varphi(y x y) .
$$

Thus

$$
\varphi(\lambda)^{2} \varphi(x y x)+\mathscr{P}(\lambda) \varphi(y x y)=\mathcal{P}(\lambda)^{2} \varphi(x) \varphi(y) \mathcal{P}(x)+\phi(\lambda) \mathcal{P}(y) \varphi(x) \varphi(y) .
$$

Multiplying the identity of Lemma 1 by $\phi(\lambda)$ and subtracting from the above we have

$$
\left(\varphi(\lambda)^{2}+\varphi(\lambda)\right)(\varphi(x y x)+\varphi(x) \varphi(y) \varphi(x))=0 .
$$

However, $\varphi(\lambda)^{2}+\varphi(\lambda)=\varphi\left(\lambda^{2}+\lambda\right)$, and since $\lambda \neq 0,1 \lambda^{2}+\lambda \neq 0$, hence by Lemma $3, \varphi\left(\lambda^{2}+\lambda\right) \neq 0$. Thus $\phi(\lambda)^{2}+\varphi(\lambda)$ is a non-zero element in the center of a prime ring $R^{\prime}$; since it is annihilated by $\varphi(x y x)+\varphi(x) \varphi(y) \varphi(x)$, it must be that $\varphi(x y x)+\varphi(x) \varphi(y) \varphi(x)=0$. This completes the proof of the lemma.

Lemma 7. For any $x, y \in R$ define $z$ by

$$
\varphi(z)=\varphi(x y x)+\varphi(x) \mathcal{P}(y) \varphi(x) \text {. Then } \mathcal{P}\left(z^{3}\right)=\varphi\left(z^{2}\right) .
$$

Proof. From Lemma 4 it follows that $[\varphi(z), \varphi(x)]=0$ and $[\varphi(z), \varphi(y)]=$ 0 . But then $\varphi[z, x]=0$ and $\varphi[z, y]=0$; consequently by Lemma 3 , $[z, x]=0$ and $[z, y]=0$. Substituting $z x$ for $x$ in Lemma 1 and repeating the argument used in the proof of Lemma 6 it can be shown that

$$
\left(\varphi\left(z^{2}-z\right)\right)(\varphi(x y x)+\phi(x) \varphi(y) \varphi(x))=0 .
$$

But then $\varphi\left(z^{2}-z\right) \varphi(z)=0$; since $z^{2}-z$ commutes with $z$, Lemma 5 implies that $\varphi\left(\left(z^{2}-z\right) z\right)=0$, that is, $\varphi\left(z^{3}\right)=\varphi\left(z^{2}\right)$. The lemma is thus proved. 
This lemma allows us to generate idempotents. It is thus natural to examine the behavior of an idempotent under $\varphi$. We do this in the next two lemmas.

Lemma 8. If $e$ is an idempotent in $R$ then for all $x R$,

$$
\mathcal{P}(e x e)=\mathcal{Q}(e) \mathcal{P}(x) \mathcal{P}(e) \text { and } \phi(x e x)=\varphi(x) \mathcal{P}(e) \varphi(x) \text {. }
$$

Proof. Since $e$ commutes with $x+e x+x e$, by applying Lemma 5 we obtain

$$
\begin{aligned}
\varphi(e x e) & =\varphi(e(x+e x+x e))=\varphi(e) \varphi(x+e x+x e) \\
& =\varphi(e)(\varphi(x)+\varphi(e) \varphi(x)+\varphi(x) \varphi(e)) .
\end{aligned}
$$

Since exe commutes with $e$, and since $\varphi(e)^{2}=\varphi\left(e^{2}\right)=\varphi(e)$, right-multiplying the relation obtained by $\phi(e)$ we have that

$$
\begin{aligned}
\varphi(e x e)=\varphi(e x e) \varphi(e) & =\phi(e)(\phi(x)+\varphi(e) \varphi(x)+\varphi(x) \varphi(e)) \varphi(e) \\
& =\phi(e) \mathcal{P}(x) \varphi(e) .
\end{aligned}
$$

The second part of the lemma follows from this and Lemma 1.

Lemma 9. If $R$ contains an idempotent $e \neq 0,1$ then $\varphi(x y x)=$ $\varphi(x) \varphi(y) \varphi(x)$ for all $x, y \in R$.

Proof. If $e$ and $f$ are any two idempotents then

$$
\begin{aligned}
& \phi((e+f) x(e+f))=\varphi(e x e)+\varphi(f x f)+\phi(e x f+f x e) \\
& \quad=\phi(e) \varphi(x) \varphi(e)+\varphi(f) \varphi(x) \varphi(f)+\phi(e) \varphi(x) \varphi(f)+\varphi(f) \varphi(x) \varphi(e)
\end{aligned}
$$

by Lemmas 8 and 2 . Thus $\varphi((e+f) x(e+f))=\varphi(e+f) \varphi(x) \varphi(e+f)$ for all $x \in R$. By the main result of Amitsur [1] and a direct verification for $2 \times 2$ matrics over $G F(2)$, in a simple ring having a non-trivial idempotent every commutator $v$ can be written as a sum of idempotents, so that $\varphi(v x v)=\varphi(v) \varphi(x) \varphi(v)$, consequently $\varphi(x v x)=\varphi(x) \varphi(v) \varphi(x)$ by Lemma 1. Now

$$
[\varphi(x y x), \varphi(z)]=\varphi[x y x, z]=\varphi(z x y x+x y x z) .
$$

But

$$
z x y x+x y x z=(x z+z x) y x+x y(x z+z x)+x(y z+z y) x .
$$

Since $y z+z y$ is a commutator,

$$
\begin{aligned}
& \varphi(x(y z+z y) x)=\varphi(x) \varphi(y z+z y) \varphi(x) \\
& \quad=\varphi(x) \varphi(y) \varphi(z) \varphi(x)+\varphi(x) \varphi(z) \varphi(y) \varphi(x) .
\end{aligned}
$$

Also, by Lemma 2, 


$$
\begin{aligned}
\mathcal{P}\{(x z+z x) y x+x y(x z+z x)\} \\
= \\
\quad \\
\quad+\phi(x) \mathcal{P}(z) \varphi(y) \varphi(y) \varphi(z) \varphi(x) .
\end{aligned}
$$

Combining all these relations we have that

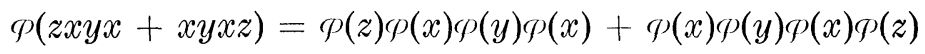

$$
\begin{aligned}
& =[\rho(x) \varphi(y) \rho(x), \varphi(z)] \text {. }
\end{aligned}
$$

As a consequence,

$$
[\mathcal{H}(x y x), \mathcal{}(z)]=[\mathcal{H}(x) \mathcal{\rho}(y) \mathcal{P}(x), \mathcal{P}(z)],
$$

and so

$$
\lambda=\mathcal{P}(x y x)+\mathcal{P}(x) \mathcal{P}(y) \mathcal{P}(x)
$$

must be in the center of $R^{\prime}$. If $\lambda=0$ we would have proved the lemma. If, on the other hand, $\lambda \neq 1$, by Lemma 6 it would follow that $\lambda=0$. Thus, for some $x, y \in R$, suppose that $\varphi(x y x)+\varphi(x) \varphi(y) \varphi(x)=1$. Then

$$
\varphi\left(x^{2} y x^{2}\right)=\varphi(x) \varphi(x y x) \varphi(x)+\mu=\varphi(x)^{2} \phi(y) \mathcal{P}(x)^{2}+\varphi(x)^{2}+\mu
$$

where $\mu$ is in the center of $R^{\prime}$. On the other hand, $\mathcal{P}\left(x^{2} y x^{2}\right)=$ $\varphi(x)^{2} \varphi(y) \varphi(x)^{2}+\rho$ where $\rho$ is in the center of $R^{\prime}$. Comparing these equalities we see that $\varphi(x)^{2}$ is in the center of $R^{\prime}$. Thus $x^{2}$ must be in the center of $R$. But then $x$ commutes with $x y+y x$; by Lemma 5 ,

$$
\begin{aligned}
\varphi(x(x y+y x)) & =\varphi(x) \varphi(x y+y x)=\varphi(x)(\varphi(x) \varphi(y)+\varphi(y) \varphi(x)) \\
& =\varphi(x)^{2} \varphi(y)+\varphi(x) \varphi(y) \varphi(x) .
\end{aligned}
$$

Since $x^{2}$ and $y$ commute, $\phi\left(x^{2} y\right)=\varphi(x)^{2} \phi(y)$. Thus by subtraction, $\mathcal{P}(x y x)=$ $\mathcal{P}(x) \phi(y) \varphi(x)$, contradicting $\varphi(x y x)+\mathcal{P}(x) \varphi(y) \varphi(x)=1$. Hence $\phi(x y x)=$ $\varphi(x) \varphi(y) \varphi(x)$ for all $x, y \in R$, proving the lemma.

We now are in a position to show that products of the form aba are preserved by $\varphi$; this will allow us to use Smiley's result thus characterizing $\varphi$. We do this in.

Lemma 10. For all $x, y \in R, \varphi(x y x)=\varphi(x) \varphi(y) \varphi(x)$.

Proof. If $R$ contains an idempotent $e \neq 0,1$ then the lemma is true as a consequence of Lemma 9. Hence we assume that $R$ has no nontrivial idempotents; since $\rho$ preserves squares and has only 0 in its kernel, $R^{\prime}$ also has no non-trivial idempotents. Let $\varphi(z)=\varphi(x y x)+\varphi(x) \varphi(y) \varphi(x)$. As we have seen in Lemma $7, \phi(z)^{3}=\varphi(z)^{2}$; thus

$$
\begin{aligned}
\varphi\left(z^{4}\right) & =\varphi(z)^{4}=\varphi(z)^{3} \varphi(z)=\varphi(z)^{2} \varphi(z) \\
& =\varphi(z)^{3}=\varphi(z)^{2}=\varphi\left(z^{2}\right),
\end{aligned}
$$


Consequently $z^{4}=z^{2}$, and so $z^{2}$ is an idempotent. But then, either $z^{2}=0$ or $z^{2}=1$. Suppose that $z^{2}=1$; then $\varphi\left(z^{2}\right)=1$. and since $\varphi(z)^{3}=\mathcal{P}(z)^{2} \varphi(z)=$ $\varphi(z)^{2}=1$, it follows that $\varphi(z)=1$, and so $z=1$.

Now let $x$ be any fixed element of $R$ and let $S^{\prime}$ be the set of all elements $r^{\prime} \in R^{\prime}$ such that $r=\mathcal{Q}(x y x)+\phi(x) \varphi(y) \varphi(x)$ for all $y \in R$. $S^{\prime}$ is clearly closed under subtraction. Also, for all $z \in R$,

$$
\left[r^{\prime}, \mathcal{P}(z)\right]=\mathscr{P}[x y x, z]+\mathcal{P}(z) \mathcal{P}(x) \mathcal{P}(y) \mathcal{P}(x)+\mathcal{P}(x) \mathcal{P}(y) \mathcal{P}(x) \varphi(z) .
$$

Since $[x y x, z]=[x, z] y x+x[y, z] x+x y[x, z]$,

$$
\begin{aligned}
& \mathscr{P}[x y x, z]=\mathcal{P}(x \mid y, z\rfloor x)+\mathcal{P}(\lfloor x, z]) \mathcal{P}(y) \mathcal{P}(x)+\mathcal{P}(x) \mathcal{P}(y) \mathscr{\mathcal { C }}[x, z] \\
& =\varphi(x[y, z] x)+\varphi(x) \varphi(z) \varphi(y) \varphi(x)+\phi(z) \varphi(x) \varphi(y) \varphi(x) \\
& +\phi(x) \varphi(y) \varphi(x) \varphi(z)+\varphi(x) \varphi(y) \mathcal{P}(z) \mathcal{P}(x)
\end{aligned}
$$

\section{from Lemma 2.}

Thus $\left[r^{\prime}, \varphi(z)\right]=\varphi(x[y, z] x)+\varphi(x) \varphi[y, z] \varphi(x)$, and so is in $S^{\prime}$. Thus $S^{\prime}$ is a Lie ideal of $R^{\prime}$. Suppose that $r, s \in S^{\prime}$. We claim that they must commute. If either is equal to 1 this of course is true. Suppose otherwise. Then $r^{2}=s^{2}=0$. If it is also true that $(r+s)^{2}=0$, then $r s+s r=0$, and $r$ and $s$ do commute. So it must be that $r+s=1$, but then trivially $r$ and $s$ commute. Thus in all cases $r s=s r$.

By a theorem of Herstein [3], since $S^{\prime}$ is a Lie ideal of $R^{\prime}$, either $S^{\prime}$ is contained in the center of $R^{\prime}$ or $S^{\prime}$ contains all commutators, except if $R^{\prime}$ is 4 -dimensional over its center. In the last instance, $R^{\prime}$ has a non-trivial idempotent, which we have ruled out. So indeed either $S^{\prime}$ is in the center of $R^{\prime}$ or contains all commutators.

However any two elements of $S^{\prime}$ commute, so the ring generated by $S^{\prime}$ is commutative; however by a result of Herstein [3], the ring generated by the commutators is all of $R^{\prime}$ unless $R^{\prime}$ is a field, (but in that case $R$ is a field, contrary to assumption). So $S^{\prime}$ can not generate all of $R^{\prime}$, whence $S^{\prime}$ is contained in the center of $R^{\prime}$.

If $S^{\prime}$ is contained in the center of $R^{\prime}$, it can have non-zero nilpotent elements. Thus $S^{\prime}$ consists only of 0 and 1 .

If the lemma is false, for some $y \in R, \varphi(x y x)=\varphi(x) \varphi(y) \varphi(x)+1$. Now $\varphi\left(x^{2} y x^{2}\right)=\varphi(x)^{2} \varphi(y) \varphi(x)^{2}+\beta$ for $\beta$ in center of $R^{\prime}$. But $\varphi\left(x^{2} y x^{2}\right)=$ $\phi(x \cdot x y x \cdot x)=\varphi(x \cdot x y x \cdot x)=\varphi(x) \varphi(x y x) \varphi(x)+\alpha$ (where $\alpha$ is in the center of $\left.R^{\prime}\right)=\varphi(x)^{2}+\varphi(x)^{2} \varphi(y) \varphi(x)^{2}+\alpha$. Comparing the two results leads to the fact that $\varphi(x)^{2}$ lies in the center of $R^{\prime}$, and so $x^{2}$ is in the center of $R$. But then $x$ commutes with $x y+y x$ and so, by Lemma 5

$$
\varphi(x(x y+y x))=\varphi(x) \varphi(x y+y x)=\varphi(x)(\varphi(x) \varphi(y)+\phi(y) \varphi(x)) .
$$

Since $x^{2}$ is in the center of $R, \mathcal{P}\left(x^{2} y\right)=\mathcal{P}(x)^{2} \mathcal{P}(y)$. The above relation 
then reduces to $\phi(x y x)=\varphi(x) \varphi(y) \varphi(x)$, contrary to the fact that $\varphi(x y x)=$ $\varphi(x) \varphi(y) \varphi(x)+1$. This contradiction leads to the proof of Lemma 10 .

From this point on the proof given by Smiley [6] can be used to show that

THEOREM 1. If $\varphi$ is an additive mapping from a simple ring $R$ onto a simple ring $R^{\prime} \neq 0$ of characteristic 2 and if $\varphi\left(x^{2}\right)=\varphi(x)^{2}$, $\varphi\left(x^{3}\right)=\varphi(x)^{3}$ for all $x \in R$ then $\phi$ is either an isomorphism or an antiisomorphism.

The following remark is in order. The assumption (ii) may be weakened to

$$
(\text { ii })^{*} \quad \varphi(x y-y x)=\varphi(x) \varphi(y)-\varphi(y) \varphi(x) .
$$

For then

$$
\begin{aligned}
\varphi(x)^{3} & +\varphi(y)^{3}+\varphi(x)^{2} \varphi(y)+\varphi(x) \varphi(y) \varphi(x)+\varphi(y) \varphi(x)^{2}+\varphi(y)^{2} \varphi(x) \\
& +\phi(y) \varphi(x) \varphi(y)+\varphi(x) \varphi(y)^{2} \\
= & \varphi\left((x+y)^{3}\right)=\varphi\left(x^{3}+y^{3}+x^{2} y+x y x+y x^{2}+y^{2} \bar{x}+y x y+x y^{2}\right) .
\end{aligned}
$$

Since

$$
\begin{aligned}
\varphi\left(x^{3}\right) & =\varphi\left(y^{3}\right), \varphi\left(y^{3}\right)=\varphi(y)^{3}, \quad \text { and } \varphi\left[x^{2}, y\right]=\left[\varphi\left(x^{2}\right), \varphi(y)\right] \\
& =\phi[x, x y+y x]=[\varphi(x), \varphi(x) \varphi(y)+\varphi(y) \varphi(x)]=\left[\varphi(x)^{2}, \varphi(y)\right] .
\end{aligned}
$$

Thus $\varphi\left(x^{2}\right)-\varphi(x)^{2}=\lambda$ is in the center of $R^{\prime}$. But then

$$
\phi(y) \varphi\left(x^{2}\right)+\phi\left(x^{2}\right) \varphi(y)=\phi(y) \varphi(x)^{2}+\varphi(x)^{2} \phi(y),
$$

and

$$
\phi(x) \varphi\left(y^{2}\right)+\varphi\left(y^{2}\right) \varphi(x)=\varphi(x) \varphi(y)^{2}+\varphi(y)^{2} \phi(x) .
$$

Substituting these in the linearized form above of $\varphi\left((x+y)^{3}\right)$, we obtain

$$
\varphi(x y x+y x y)=\varphi(x) \varphi(y) \varphi(x)+\varphi(y) \varphi(x) \varphi(y) .
$$

If the center of $R^{\prime}$ consists only of 0 then from above, $\varphi\left(x^{2}\right)=\varphi(x)^{2}$ follows and so the theorem would follow from Theorem 1. Suppose that $R^{\prime}$ has a non-trivial center. Then $1 \in R^{\prime}$. Let $a \in R$ be such that $\varphi(a)=1$. Thus for all $x \in R, \varphi(a x+x a)=0$. Let $K=\{u \in R \mid \varphi(u)=0\} . \quad K$ is a Lie ideal of $R$. If $R$ is 4-dimensional over its center, then $R$ has a unit element and by the remark made earlier in the paper this would imply that $\varphi\left(x^{2}\right)=\varphi(x)^{2}$ for all $x \in R$, which by Theorem 1 would imply the theorem. If $R$ is more than 4 -dimensional over its center by the main result of [3], since $K$ is a Lie ideal of $R$, either $K$ if contained in $Z$ the center of $R$ or $K \supset[R, R]$.

In the first case, since $a x+x a \in K$ for all $x \in R$, if $w=a x+x a \neq 0$, 
then $Z \neq 0$, and so $R$ has a unit element and the result would follow. If $a x+x a=0$ then again $0 \neq a \in Z$ and the result follows.

So suppose $K \supset[R, R]$. Thus $R^{\prime}$ is a field. But then as we have previously seen in [2], $\varphi\left(x^{2} y+y^{2} x\right)=\varphi(x) \varphi(y) \varphi(x+y)$. Putting $x=a$ where $\varphi(a)=1$, we obtain that $\varphi\left(a^{2} y+y^{2} a\right)=\varphi(y)+\varphi(y)^{2}$. Let $t \in R$, such that $\varphi(t)=0$. Then $\varphi\left(t^{2} y+y^{2} t\right)=0$. Also $\varphi(a+t)=1$, so

$$
\begin{aligned}
& \varphi\left((a+t)^{2} y+y^{2}(a+t)\right)=\varphi(y)+\varphi(y)^{2} \\
& \quad=\varphi\left(a^{2} y+y^{2} a+(a t+t a) y+t^{2} y+y^{2} t\right) .
\end{aligned}
$$

Using the results obtained above this relation simplifies to $\varphi((a t+t a) y)=0$ for all $y$. But then $\varphi(R(a t+t a) R)=0$; if $a t+t a \neq 0$, by the simplicity of $R$ this yields $R^{\prime}=\phi(R)=0$. So we must assume that $a t=t a$ for all $t$ such that $\varphi(t)=0$. In particular $a$ must commute with all commutators. Thus $a(a x+x a)=(a x+x a) a$, and so $a^{2}$ is in the center. If $a^{2}=0$ then $a^{3}=0$ and so $0=\varphi\left(a^{3}\right)=\varphi(a)^{3}=1$, a contradiction. Thus $a^{2} \neq 0$ and so $Z \neq 0$. Thus $1 \in Z$ and so $\varphi$ must preserve squares. Thus using Theorem 1 again we would be done. Thus we have seen that at all times (i), (ii) and (iii) are satisfied and the conclusion of Theorem 1 still holds. This establishes

THEOREM 2. If $\phi$ is an additive mapping from a simple ring $R$ onto a simple ring $R^{\prime} \neq 0$ of characteristic 2 and if $\varphi(x y-y x)=$ $\varphi(x) \varphi(y)-\varphi(y) \varphi(x)$ and $\varphi\left(x^{3}\right)=\varphi(x)^{3}$ for all $x, y \in R$ then $\varphi$ is either an isomorphism or an anti-isomorphism.

\section{BIBLIOGRAPHY}

1. S. Amitsur, Invariant submodules of simple rings, proc. A. M. S., 7 (1951), 987-989.

2. I. N. Herstein, Jordan homomorphisms, Trans. A. M. S., 81 (1956), 331-341.

3. - On the Lie and Jordan rings of a simple associative ring, Amer. J. Math., 77 (1955), 279-285.

4. L. K. Hua, On the automorphisms of a s-field, Proc. Nat. Acad. Science (USA), 35 (1949), 386-389.

5. N. Jacobson, Classes of restricted Lie algebras, Amer. Jour. Math., 63 (1941), 481-515.

6. M. F. Smiley, Jordan homomorphisms onto prime rings, Trans. A. M. S., 84 (1957), 426-429.

CORNELL UNIVERSITY

AND

OHIo StATE UNIVERSity 


\section{PACIFIC JOURNAL OF MATHEMATICS}

\section{EDITORS}

\author{
David Gilbarg \\ Stanford University \\ Stanford, California \\ F. H. Brownell \\ University of Washington \\ Seattle 5 , Washington
}

\author{
A. L. Whiteman \\ University of Southern California \\ Los Angeles 7, California \\ L. J. PAIGE \\ University of California \\ Los Angeles 24, California
}

\section{ASSOCIATE EDITORS}

\author{
E. F. BECKENBACH \\ T. M. CHERRY \\ D. DERRY
}
E. HEWITT
A. HORN
L. NACHBIN
M. OHTSUKA
H. L. ROYDEN
M. M. SCHIFFER

E. SPANIER

E. G. STRAUS

F. WOLF

\section{SUPPORTING INSTITUTIONS}

\author{
UNIVERSITY OF BRITISH COLUMBIA \\ CALIFORNIA INSTITUTE OF TECHNOLOGY \\ UNIVERSITY OF CALIFORNIA \\ MONTANA STATE UNIVERSITY \\ UNIVERSITY OF NEVADA \\ NEW MEXICO STATE UNIVERSITY \\ OREGON STATE COLLEGE \\ UNIVERSITY OF OREGON \\ OSAKA UNIVERSITY \\ UNIVERSITY OF SOUTHERN CALIFORNIA
}

\author{
STANFORD UNIVERSITY \\ UNIVERSITY OF TOKYO \\ UNIVERSITY OF UTAH \\ WASHINGTON STATE COLLEGE \\ UNIVERSITY OF WASHINGTON

AMERICAN MATHEMATICAL SOCIETY
CALIFORNIA RESEARCH CORPORATION
HUGHES AIRCRAFT COMPANY
SPACE TECHNOLOGY LABORATORIES
NAVAL ORDNANCE TEST STATION

Mathematical papers intended for publication in the Pacific Journal of Mathematics should be typewritten (double spaced), and the author should keep a complete copy. Manuscripts may be sent to any one of the four editors. All other communications to the editors should be addressed to the managing editor, L. J. Paige at the University of California, Los Angeles 24, California. ..

50 reprints per author of each article are furnished free of charge; additional copies may be obtained at cost in multiples of 50 .

The Pacific Journal of Mathematics is published quarterly, in March, June, September, and December. The price per volume (4 numbers) is $\$ 12.00$; single issues, $\$ 3.50$. Back numbers are available. Special price to individual faculty members of supporting institutions and to individual members of the American Mathematical Society: $\$ 4.00$ per volume; single issues, $\$ 1.25$.

Subscriptions, orders for back numbers, and changes of address should be sent to Pacific Journal of Mathematics, 2120 Oxford Street, Berkeley 4, California.

Printed at Kokusai Bunken Insatsusha (International Academic Printing Co., Ltd.), No. $6_{4}$ 2-chome, Fujimi-cho, Chiyoda-ku, Tokyo, Japan.

PUBLISHED BY PACIFIC JOURNAL OF MATHEMATICS, A NON-PROFIT CORPORATION

The Supporting Institutions listed above contribute to the cost of publication of this Journăl, but they are not owners or publishers and have no responsibility for its content or policies. 


\section{Pacific Journal of Mathematics}

\section{Vol. 10, No. $3 \quad$ November, 1960}

Glen Earl Baxter, An analytic problem whose solution follows from a simple

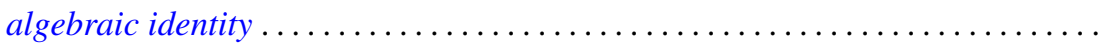

Leonard D. Berkovitz and Melvin Dresher, A multimove infinite game with linear payoff. .

Earl Robert Berkson, Sequel to a paper of A. E. Taylor ......................

Gerald Berman and Robert Jerome Silverman, Embedding of algebraic systems.... 767

Peter Crawley, Lattices whose congruences form a boolean algebra . . . . . ...... 777

Robert E. Edwards, Integral bases in inductive limit spaces . . . . . . . . . . . . . . .

Daniel T. Finkbeiner, II, Irreducible congruence relations on lattices . . . . . . . . . .

William James Firey, Isoperimetric ratios of Reuleaux polygons . . . . . . . . . . . 787

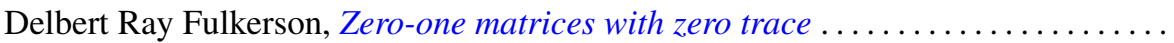

Leon W. Green, A sphere characterization related to Blaschke's conjecture........

Israel (Yitzchak) Nathan Herstein and Erwin Kleinfeld, Lie mappings in

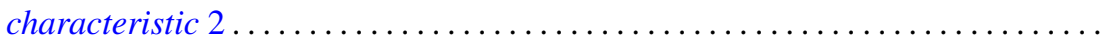

Charles Ray Hobby, A characteristic subgroup of a $p$-group .................

R. K. Juberg, On the Dirichlet problem for certain higher order parabolic

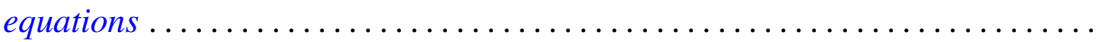

Melvin Katz, Infinitely repeatable games ......................

Emma Lehmer, On Jacobi functions . . . . . . . . . . . . . . . . . . . . . . . . .

D. H. Lehmer, Power character matrices

Henry B. Mann, A refinement of the fundamental theorem on the density of the sum of two sets of integers.

Marvin David Marcus and Roy Westwick, Linear maps on skew symmetric matrices: the invariance of elementary symmetric functions . .

Richard Dean Mayer and Richard Scott Pierce, Boolean algebras with ordered

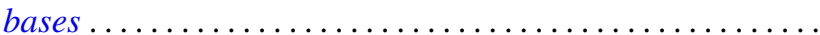

Trevor James McMinn, On the line segments of a convex surface in $E_{3} \ldots$

Frank Albert Raymond, The end point compactification of manifolds ..

Edgar Reich and S. E. Warschawski, On canonical conformal maps of regions of arbitrary connectivity

Marvin Rosenblum, The absolute continuity of Toeplitz's matrices...

Lee Albert Rubel, Maximal means and Tauberian theorems . .

Helmut Heinrich Schaefer, Some spectral properties of positive linear operators

Jeremiah Milton Stark, Minimum problems in the theory of pseudo-conformal transformations and their application to estimation of the curvature of the invariant metric.

Robert Steinberg, The simplicity of certain groups ...

Hisahiro Tamano, On paracompactness. .

Angus E. Taylor, Mittag-Leffler expansions and spectral theory .

Marion Franklin Tinsley, Permanents of cyclic matrices ...... . 